\title{
Modeling Immune-Mediated Activations and Interactions in Breast Cancer Progression
}

\author{
Kodwo Annan \\ School of Science and Technology, Georgia Gwinnett College, Lawrenceville, USA \\ Email address: \\ kannan@ggc.edu \\ To cite this article: \\ Kodwo Annan. Modeling Immune-Mediated Activations and Interactions in Breast Cancer Progression. International Journal of Systems \\ Science and Applied Mathematics. Vol. 4, No. 1, 2019, pp. 1-12. doi: 10.11648/j.ijssam.20190401.11
}

Received: January 31, 2019; Accepted: March 18, 2019; Published: April 3, 2019

\begin{abstract}
Mathematical model that allowed qualitative and quantitative description of the interactions between the host immune system, breast cancer cells, and a cancer vaccine was presented with a system of differential equations. Key immune components used in the vaccine were cytotoxic T lymphocytes (CTLs), macrophages, Natural Killer (NK) and helper T cells. The parameters of the model were based on experimental and clinical results from published articles. MATLAB software tool was used to generate data from the model and results were analyzed and discussed. Findings supported clinical studies that maximum immune activation was needed to reduce the cancer cells. Thus, for a given breast cancer growth rate, there was an optimal activation that maximized the response of the immune system. It was also observed that given a sufficiently high rate of CTLs, natural killer, or helper T cells infiltration resulted in significant tumor elimination. However, varying CTLs and Macrophages activation rates caused a chaotic behavior of the tumor. Thus, optimizing large M1:M2 ratios verses large/small numbers of tumor-infiltrating macrophages on long term patient survival were necessary in improving breast cancer therapies.
\end{abstract}

Keywords: Breast Cancer, Immune System, Mathematical Model

\section{Introduction}

Diagnosis and management of breast cancer has undergone many changes over the past few decades. While mammographic screening is still the primary method for diagnosing breast cancer, technologies such as magnetic resonance imaging (MRI) and positron emission tomography (PET) scans are finding some cancers missed by mammograms. Furthermore, microarray technology shows promise for predicting aggressiveness of cancer and assisting physicians in determining the best type of therapy a patient needs. Despite these innovations in diagnosis and concurrent improvements in treatment, many women still die of breast cancer. It remains the commonest malignancy in women in United States with an estimated 268,670 new cases of invasive breast cancer [1] and about 40,920 women are expected to die in 2019 from the disease [2]. The complexity of the disease morphology, biological heterogeneity, tendency for the disease to become resistant to chemotherapy, and several molecular pathways of the disease are some of the reasons given for the high incidence and mortality rates.
The role of the immune system in controlling breast tumor progression has been studied by many authors [2-6] and significant development has been made in the treatment of breast cancer, especially with the growing use of cancer vaccines [7-9]. The breast cancer tumor microenvironment is composed of a variety of immune cells that can control or capture the malignant progression. Research has shown that immune system plays an antagonistic role in the tumoral environment of breast cancer. Even though the immune's primary goal of preventing tumor formation is through immune surveillance, some immune cells promote alternate inflammatory pathways that suppress adaptive immunity and create a state of immunotolerance [11-13]. Immune infiltrates in breast cancer are mainly composed of $\mathrm{T}$ lymphocytes $(\approx 75 \%)$, together with B lymphocytes $(<20 \%)$, monocytes $(<10 \%)$, and natural killer cells $(<5 \%)[11,13]$. The T CD3+ lymphocytes are divided into CD8+ and CD4+ T helper (Th) cells and $\mathrm{CD} 4+$ regulatory $\mathrm{T}$ cells (Treg). The CD4+ lymphocytes are composed of all Th subsets, with a mixture of activating and suppressive activities [11]. Although studies have stated diversity in the tumoral immune microenvironment of breast cancer, there has not been any 
indication of other immune cell subsets with higher prognosis indicator than tumor-infiltrative lymphocytes (TILs) level.

The association between tumor-infiltrative lymphocytes (TILs) and prognosis in primary breast carcinoma has been studied [4, 14-19]. TILs have been associated with poorprognosis clinicopathologic characteristics, including estrogen receptor (ER) negativity, higher tumor grade, high levels of Ki-67, larger tumor size, and positive lymph nodes [6, 20-23]. Despite this, high TILs level as an independent indicator of good prognosis has been validated in large cohorts of patients. To mention a few, a high level of TILs is associated with improved distant disease-free survival, disease-free survival (DFS), and overall survival (OS) $[6,14$, 24-28]. The promising evidence supporting the prognostic and predictive role of TILs has elicited the standardization of TIL evaluation in breast cancer [19].

Currently, one approach in combating breast cancer progression is to use cancer vaccine that triggers the patient's immune system to identify the foreign antigens by stimulating cytotoxic T lymphocytes (CTL) [9-10, 31-33]. The vaccination components consist of lymphocytes, which include: helper $\mathrm{T}$ lymphocytes (Th), dendritic cells (DC), macrophages, or reprogrammed oncolytic viruses [35-37]. Such vaccines are known to help prevent cancer growth through stimulation of the patient's immune system or by directly attacking a cancer growth [8, 33]. As therapeutic agents, the immune system act as modulators from early evolution to metastatic stage and also affect tumor response to chemotherapy. However, researchers have found many issues to the vaccine development process as it displays the ability of antigen mimicry, a process by which tumor cells produce antigens with specific patterns of the host that can help cancer evade immune processing and development. Tumor antigen imitation with self-antigen occurs since tumor-specific antigens (TSA) and tumor-associated (TAA) antigens are either mutated or overexpressed self-proteins, respectively (P53 and CEA). In addition, immune microenvironment is not static. It evolves within the tumor and changes with therapeutic intervention.

Thus, more studies are needed to better understand these findings. As our understanding of the relationship between breast cancer morphology and immunity improves, it should provide new or effective progress in immunotherapy for breast cancer patients. The main issue is that it is virtually impossible to asses the impact of every particular molecular event using classical molecular biological methodology due to the complexity of the immune systems interaction with the cancer tumor. As a result, most experimental and clinical studies have focused on the role played by cytotoxic $\mathrm{T}$ cells in tumor elimination and neglected roles played by helper $\mathrm{T}$ cells [38-39, 42], dendritic cells [40-41], or macrophages [38-42]. To understand how the immune system works to eradicate breast cancer, it is necessary to identify its key components.

The Immune Microenvironment: $\mathrm{T}$ cells are one of the most important components of the immune system in the fight against any cancer. They originate from pluripotent haematopoietic stem cells in the bone marrow which migrate to the thymus where they mature into naive $\mathrm{T}$ cells. The naive $\mathrm{T}$ cells move to the lymph nodes where they become activated on contact with their cognate antigens. Activated $\mathrm{T}$ cells proliferate rapidly to produce a substantial army of antigen-specific $\mathrm{T}$ cells. These short lived $\mathrm{T}$ cells are then transported through the blood vessels to the tumor where they bind to and kill infected cells and also produce cytokines that recruit other immune cells to the tumor. This process continues until either the tumor has been removed or the tumor adapts to, and evades the targeting $\mathrm{T}$ cells [43]. There are two populations of $\mathrm{T}$ cells, helper and cytotoxic, which are distinguished by their expression of CD4 and CD8 proteins, respectively. Helper $\mathrm{T}$ cells are initiated by antigen presented with a major histocompatibility complex (MHC) class II molecule. Cytotoxic $\mathrm{T}$ cells are activated by antigen existing with an MHC class I molecule. Once activated the helper and cytotoxic $\mathrm{T}$ cells perform complementary functions to eliminate the tumor. In addition, the Helper $\mathrm{T}$ cells distinguish into subgroups categorized by the specific cytokines that they produce. In this way, they regulate multiple aspects of an immune response such as promoting proliferation of cytotoxic $\mathrm{T}$ cells, recruiting and promoting cells of the innate immune response, and controlling levels of inflammation at the tumor environment [44]. Therefore, the balance between the different $\mathrm{T}$ cell subsets is determinant for efficient antitumor activity.

Macrophages are the most versatile types of immune cells and one of the key regulators of breast cancer immunotherapy. They can display different phenotypes, in response to the type, concentration and longevity of exposure to stimulating agents [45]. The two extreme macrophages phenotypes are represented by the M1 and M2 cells. M1 cells are pro-inflammatory and may be induced by various endoand exo-genous stimuli e.g. IFN- $\gamma$ lipopolysaccharide, myramildipeptid. M2 cells do not have pro-inflammatory properties and may be induced by IL-4, IL-13, IL-10, TGFbeta or glucocorticoids which directly affects cancer cells. While it is widely accepted that the activated M1 cells have anti-tumor properties and the alternatively activated M2 cells have pro-tumor properties, many macrophages inside the tumor microenvironment have markers characterizing mixed phenotypes [46-47]. Therefore, in spite of the fact that macrophages often constitute between 50 to 80 percent of the most common cell types in breast tumors [47], their plasticity makes it difficult to fully understand their pro-tumor/antitumor roles, as well as their pro-viral/anti-viral roles. As recently emphasized in Denton et al., (2016), the macrophages can support these therapies through the suppression of the anti-viral immune response, in the case of M2 while they may impede oncolytic therapies through the promotion of an anti-viral immune response that leads to viral clearance, but may also enhance the virus-mediated activation of the anti-tumor immune response (in the case of M1), [48].

Dendritic cells (DCs) are a heterogeneous group of antigen-presenting cells (APCs) with two major subsets, 
conventional DCs (cDCs) and plasmacytoid DCs (pDCs). In vitro and ex vivo observations suggest that DCs play a crucial role in the link between innate and adaptive immunity under inflammatory conditions but also induce immunological tolerance to maintain immune homeostasis under steady-state conditions. The communication between the innate and adaptive systems is carried out by cytokines that bind to cells, and by cell-cell interactions between dendritic cells and lymphocytes (e.g. T-cells) in lymph nodes. This interaction is so crucial that the adaptive response cannot occur without an innate immune system.

Natural killer (NK) cells are a subset of cytotoxic lymphocyte that functions predominantly in the innate immune response. Evidence suggests that NK cells play an important role in the initial and developed stages of breast cancer [49]. Cancer cells are killed by releasing small cytoplasmic granules of proteins containing perforin and granzyme that cause the formation of pores in the plasma membrane of the target cell leading it to die by apoptosis [50]. NK cells have been shown to secrete Interferon gamma $(\mathrm{IFN}-\gamma)$ in regions of breast cancer sites [49-51]. Consequently, IFN $-\gamma$ causes target cells to begin upregulating cancer specific MHC class I molecules, possibly as a result of the switch by cells to instigate expression of the immunoproteosome. Thus, these results provide a possible mechanism by which $\mathrm{NK}$ cells magnify the anti-cancer response through stimulation of aberrant cells to initiate antigens recognized by CTLs. Studies have indicated that NK cells are frequently deficient or dysfunctional in patients with breast cancer malignancy, indicating that this may be a key factor in cancer immunoevasion and progression [52]. Therefore, it is imperative that our understanding of immune system interaction should include NK cell regulation and adaptation.

Given the current expanding knowledge of the immune microenvironment and its interaction with breast cancer cells, work needs to be done to explore the potential and optimization of helper T cells, macrophages, and NK cells in addition to the cytotoxic $\mathrm{T}$ cells in the next advancement of the diseases' treatment. Nevertheless, clinical and experimental studies fall short of incorporating these key immune components in the comprehensive study of breast cancer progression and treatment. In a cooperative effort with clinicians and research oncologists, however, there exists an array of mathematical models on breast cancer progression and treatment. Most of the proposed models typically does not involve immune system or were over simplified by targeting only the CTLs cells [57-63]. Mathematical models must focus on mimicking the most important elements of the multifaceted process of the disease [53-60]. When data is generated, these models provide insight and validity to a complex biological system for clinical research without the utilization of human or animal models, entirely bypassing ethics boards completely.

In this study, a mathematical modeling approach incorporating breast cancer cells interactions with helper $\mathrm{T}$, CTL, macrophages, and NK cells is developed. The model uses frameworks of differential equations that allow qualitative and quantitative description of the interactions between the host immune system and the breast cancer cells. Data generated from the model would help examine the impact of each of the key components on the cancer cells and the different effects of immunosuppression at various stages of tumor development. As clinical studies have shown that enhanced immune activation correlates with patient outcomes $[29,36,47]$, we focus on understanding where in parametric space are these activations optimized. Thus, the goal of this paper is to use mathematical modeling approach to help gain more insight of immune macroenvironment and breat tumorinfiltrative lymphocytes and to determine which epitopes produce a strong immune response.

The rest of the paper is arranged as follows: Model assumptions and mathematical equationa are formulated in section 2. Parameter estimations and model findings are discussed in sections 3 and 4 respectively. Therapeutic implications and conclusion are summarized in section 5 .

\section{Model Assumptions and Mechanisms of Cancer-Immunity Interaction}

We state the cancer-immunity mechanisms assumptions and develop a mathematical model of tumor dynamics in response to an immune vaccine activation in this section.

\subsection{Model's Assumptions}

The biological assumptions taken into consideration during the model formulation are as follows:

(1) With the exception of some other minor contributors, four types of immune cells - T-cell, NK cells, helper T cells, and macrophages are considered to play a significant role in fighting against breast cancer cells. NK cells and CTLs can kill tumor cells [67-69] and the activation of cell-mediated immunity is regulated by macrophages and others [69].

(2) The $T$ cells are abundant in their naïve stage and differentiate into CD4, CD8, and CD4 regulatory cells through simplification of the maturation process in the thymus. This model assumes a linear transitional state from the naïve to mature states.

(3) The interactions between the cancer and immune cells depend on the functioning cancer vascular system. In addition to supplying the cancer with blood, nutrients, oxygen, and removing waste, the vascular system (VS) also supplies immune effector cells with a means to infiltrate and attack cancer cells within the cancer bed.

(4) The cancer cells grow logistically in the absence of an immune system response.

(5) Cancer cells have the potential to engender cytocidal activity in previously naïve and non-cytotoxic cells.

(6) As part of the innate immune system, Natural Killer cells are always present and active in the model, even in the absence of cancer cells

(7) The Interactions between breast cancer and immune 
cells depend on a functioning cancer vascular system.

Figure 1 illustrates the schematic interactions between the cancer cells and the key elements of the immune system selected for our model. Also, the variables and parameters defined in our model equations and their values are presented in Tables 1 and 2 respectively.

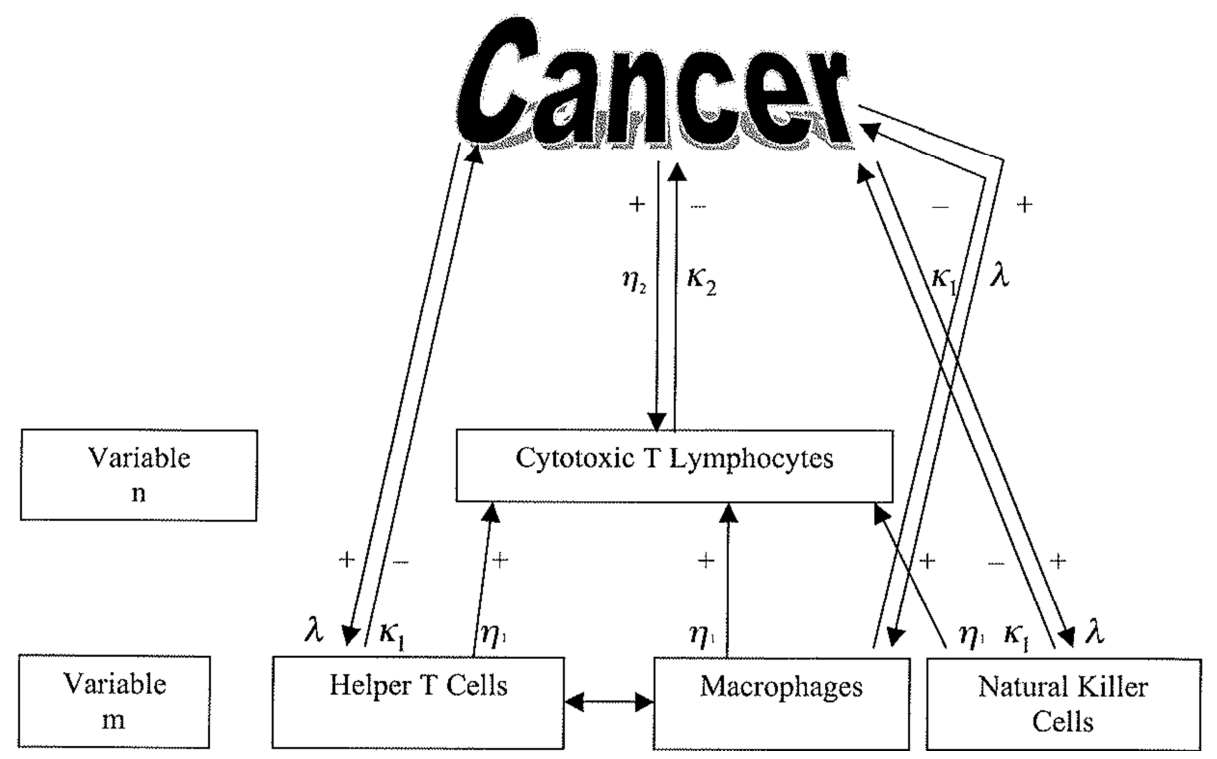

Figure 1. Shows the importance of each of the four key immune components and their interaction with the cancer cells.

Table 1. Dependent variables and cancer parameters used in our model equations.

\begin{tabular}{|c|c|}
\hline Symbol & Definition \\
\hline$c(t)$ & Mass, in grams, at time $t$ of the cancer cells \\
\hline$c_{v}(t)$ & Mass, in grams, at time $t$ of immature Vascular Systems within the cancer \\
\hline$c_{L}(t)$ & Length of mature (existing) cancer microvessels \\
\hline$v(t)$ & Cancer perfusion length density given by $v=c_{L} / c$; \\
\hline$\varphi(v)$ & Per capita growth rate function of cancer cells \\
\hline$m$ & Stimulatory immune cells capable of attacking cancer cells \\
\hline$n$ & CTL cells \\
\hline$k_{1}$ & Rate at which macrophages, NK, and T-helper cells kill cancer cells \\
\hline$k_{2}$ & Rate at which CTLs kill cancer cells \\
\hline$\varphi(v)$ & Per capita growth rate of a single cell type as a function of blood supply \\
\hline$C_{o_{2}}$ & Partial pressure of cancer oxygen measured in $\mathrm{mmHg}$ \\
\hline$C_{r}$ & Maximum cell reproduction \\
\hline$C_{m}$ & Mortality rates \\
\hline$\gamma_{1}$ & Effect of oxygen sensitivity on parenchyma reproduction rate \\
\hline$\gamma_{2}$ & Oxygen sensitivity of the parenchyma mortality \\
\hline$k$ & How quickly the cancer oxygen partial pressure response to changes in perfusion \\
\hline$h(v)$ & The angiogenesis signal produced by parenchyma cells \\
\hline$r$ & Angiogenesis signal produced by cancer cells \\
\hline$\xi$ & How angiogenesis signal production is affected by the changes in oxygen supply \\
\hline$\beta$ & Both the VEC maturation and death rates \\
\hline$\gamma c_{v}$ & Rate at which new microvessels arise from activated VECs \\
\hline$\delta$ & Basic microvessel remodeling rate \\
\hline$\lambda_{a}$ & Rate at which tumor cells activate macrophages, $\mathrm{NK}$ and helper $\mathrm{T}$ cells \\
\hline$\lambda_{i}$ & Per capita inactivation rate of macrophages NK and helper T cells \\
\hline$\eta_{1}$ & Rates at which macrophages, NK and helper T cells activate CTLs \\
\hline$\eta_{2}$ & Rate of CTL activation \\
\hline$\mu_{2}(c) n$ & The suppression rate of adaptive immune cells as a function of cancer mass \\
\hline$b$ & sensitivity of the suppression mechanism to the presence of cancer cells \\
\hline$c_{s}$ & base level of CTL suppression \\
\hline
\end{tabular}

\subsection{Breast Cancer Cells Model}

Let $c(t)$ and $c_{v}(t)$ be the mass, in grams, at time $t$ of the cancer cells and the immature VSs within the cancer respectively. Let $c_{L}(t)$ represents the length of mature (existing) cancer microvessels. Then, the normal growth rate of a single cancer cell type in a vascularized cancer 
is represented by:

$$
\frac{d c}{d t}=\varphi(v) c-c_{L}\left(k_{1} m+k_{2} n\right) c .
$$

The variable $v(t)$ represents cancer perfusion length density given by $v=c_{L} / c ; \varphi(v)$ express per capita growth rate function of cancer cells; $m$ is the stimulatory immune cells capable of attacking cancer cells as well as activating CTLs; $n$ represents CTLs; $k_{1}$ express the rate at which macrophages, $\mathrm{NK}$, and THCs kill cancer cells; $k_{2}$ is the rate at which CTLs kill cancer cells. The equation includes the cancer cell death rate as a result of their interactions with two groups of immune cells. The first group consists of macrophages, NK and helper $\mathrm{T}$ cells and the second group consists solely of CTLs (see Figure 1). The rates $k_{1}$ and $k_{2}$ depend on the total length of existing cancer microvessels, $c_{L}(t)$. Also, we have scaled $c_{L}(t)$ in such a way that one unit of microvessels is equivalent to the mean length of microvessels in one gram of unaffected tissue.

The function $\varphi(v)$ which expresses per capita growth rate of a single cell type as a function of blood supply is modeled as

$$
\varphi(v)=\frac{C_{r} C_{o_{2}}^{p}}{\gamma_{1}^{p}+C_{o_{2}}^{p}}-C_{m}\left(1-\frac{C_{o_{2}}^{q}}{\gamma_{2}^{q}+C_{o_{2}}^{q}}\right)
$$

Here, $C_{\mathrm{O}_{2}}^{p}$ denotes partial pressure of cancer oxygen measured in mmHg; the parameters $C_{r}$ and $C_{m}$ represent the maximum cell reproduction and mortality rates, respectively. $\gamma_{1}$ measures the effect of oxygen sensitivity on parenchyma reproduction rate while $\gamma_{2}$ reflects the oxygen sensitivity of the parenchyma mortality. Thus, the first term on the right-hand-side of equation (2) expresses the parenchyma growth rate while the second term represents the parenchyma death.

In this model, we assume that the dependence of oxygen concentration on vascularization is

$$
C_{o_{2}}^{p}=\frac{C_{\max } v}{k+v}
$$

where the maximum possible oxygen partial pressure cancer in the patient's tissues is denoted by $C_{\max }=95 \mathrm{mmHg}$ under normal conditions $[7,70]$. The parameter $k$ measures how quickly the cancer oxygen partial pressure response to changes in perfusion and is given by $[7,70]$ as 1.375 perfusion units. Therefore, the function $\varphi$ is fully characterized in term of $v$.

\subsection{Vascular Endothelial Cells Model}

The equation for the immature vascular endothelial cells (VEC) dynamics is given by:

$$
\frac{d c_{v}}{d t}=(\alpha h(v)-\beta) c_{v}
$$

The term $\alpha h(v)$ represents the proliferation rate of VEC precursors while the parameter $\alpha$ measures the VEC precursor response to breast cancer angiogenesis factors (CAF). The function $h(v)$ which describes the angiogenesis signal produced by parenchyma cells is modeled by:

$$
h(v)=r C_{o_{2}} e^{-\xi C_{o_{2}}}
$$

where the parameter $r$ measures the angiogenesis signal produced by cancer cells and $\xi$ expresses how angiogenesis signal production is affected by the changes in oxygen supply. The vanishing rate of VECs, either by dying or becoming mature microvessels, is denoted by $\beta c_{v}$. The parameter $\beta$ represents both the VEC maturation and death rates, and therefore can be referred to as per capita VEC disappearance rate.

\subsection{Microvessels Model}

The remodeling rate of mature microvessels is expressed by

$$
\frac{d c_{L}}{d t}=\gamma c_{v}-\delta v c_{L}
$$

The term $\gamma c_{v}$ expresses the rate at which new microvessels arise from activated VECs. The second term on the right in equation (6) signifies mature microvessel remodeling rate. Parameter $\delta$ represents the basic microvessel remodeling rate.

\subsection{Macrophages, Natural Killer, and Helper T Cells Model}

The activation and inactivation of macrophages, NK, and helper $\mathrm{T}$ cells in response to the presence of a cancer is modeled by:

$$
\frac{d m}{d t}=\lambda_{a} c m-\lambda_{i} m .
$$

The first term characterizes the activation of macrophages, NK and helper $\mathrm{T}$ cells. The activation rate is proportional to the number of interactions between cancer and immune cells. Parameter $\lambda_{a}$ is the rate at which tumor cells activate macrophages, NK and helper $\mathrm{T}$ cells. The second term expresses the inactivation rate of macrophages, NK and 
helper $\mathrm{T}$ cells. Parameter $\lambda_{i}$ represents the per capita inactivation rate of macrophages NK and helper T cells.

\subsection{Cytotoxic T-Lymphocytes Model}

The activation and suppression of CTLs is described by equation (8),

$$
\frac{d n}{d t}=\eta_{1} m n+\eta_{2} c n-\mu_{2}(c) n .
$$

The first term symbolizes the rate of activation of CTLs by macrophages, NK and helper T cells.

Parameter $\eta_{1}$ represents rates at which macrophages, NK and helper T cells activate CTLs. This term uses mass action because the immune-dependent activation rate of CTLs is assumed to be proportional to the interaction rate between CTLs and macrophages, NK and helper $\mathrm{T}$ cells, with proportionality $\eta_{1}$. The second term expresses the activation rate of CTLs as a result of interactions with cancer cells. The rate of CTL activation, $\eta_{2}$, is proportional to the number of parenchyma cells in the cancer. The final term $\mu_{2}(c) n$ corresponds to the suppression rate of adaptive immune cells as a function of cancer mass where

$$
\mu_{2}(c)=a e^{-b c}+c_{s}
$$

expresses the suppression rate of CTLs. The suppression is modeled as a decreasing function of $c$ since as a cancer increases in mass, we expect CTL suppression to decrease in order to maintain a strong immune response. However, once the cancer is destroyed, a large percentage of activated CTLs will remain in circulation. Therefore, suppression should increase in order to down-regulate the activity of CTLs. Parameter $a+c_{s}$ designates the maximum suppression rate of CTLs in the absence of a cancer cells. Parameter $b$ represents the sensitivity of the suppression mechanism to the presence of cancer cells, and the base level of CTL suppression is symbolized by parameter $c_{s}$.

\section{Parameter Estimation}

Prior to analyzing the behavior of the model, we estimate parameter values. Many of the biological aspects of this model are currently unavailable in literature or still not verified. However, rough approximations can be made for most of the parameters. For cancer and vascularization parameters defined in Table 1, we selected values that produced a viable cancer according to research done by [7, $41,70,71]$. We then made estimates of the appropriate order of magnitude for all immune parameters. Reasonable values for parameters $\lambda_{a}, \eta_{1}$, and $\eta_{2}$, the activation rates of immune cells, was made the same order of magnitude as the values used by $[7,70]$ for the reproduction rate of cancer cells. Thus, these parameters ranged from 0.01 to 0.1 . The inactivation rate of macrophages, $\mathrm{NK}$ and helper $\mathrm{T}$ cells also had values similar to the death rate of cancer cells. Specifically, values for parameter $\lambda_{i}$ ranged from 0.01 to 0.05. Parameter $b$ the sensitivity of the suppression mechanism in the presence of a cancer was estimated to be fairly large, ranging from 1.0 to 2.0 , in order to allow an immediate immune response in the presence of a cancer. The base CTL suppression rate $\left(c_{s}\right)$, on the other hand, should be small, ranging roughly between 0.001 and 0.01 . This range guaranteed a slow disappearance of CTLs in the presence of a cancer. Parameter $a$ corresponds to maximum CTL suppression in the absence of a cancer and thus should range from 0.05 to 0.5 in order to ensure that CTLs are sufficiently down regulated when no cancer is present. Finally, it was estimated that the parameters $k_{1}$ and $k_{2}$, the rates that our two groups of immune cells kill cancer, should range between 0.1 and 1 . This range accounts for the increased rate of cancer cell death as a result of interactions with immune cells. Table 2 summarizes the parameter estimates used in this paper.

\begin{tabular}{|c|c|c|c|c|c|c|c|}
\hline Symbol & Value & Units & Refs. & Symbols & Value & Units & Refs. \\
\hline$c(t)$ & 0.01 & $\mathrm{mg}$ & {$[8,64]$} & $\beta$ & 0.04 & Per day & {$[8]$} \\
\hline$c_{v}(t)$ & 0.001 & $\mathrm{mg}$ & {$[8,64]$} & $r$ & 0.28 & - & [65] \\
\hline$c_{L}(t)$ & 0.0 & MU & {$[8,64]$} & $\xi$ & 0.06 & - & {$[8,64]$} \\
\hline$v(t)$ & - & $\mathrm{MU} / \mathrm{gm}$ & & $\gamma$ & 3.0 & - & [64] \\
\hline$m$ & 0.001 & $\mathrm{mg}$ & {$[8,64]$} & $\delta$ & 0.004 & Per day & [66] \\
\hline$n$ & 0.0005 & $\mathrm{mg}$ & {$[8,64]$} & $\lambda_{a}$ & 0.05 & Per day & [66] \\
\hline$k_{1}$ & $0.1-1.0$ & Per day & [65] & $\lambda_{i}$ & 0.02 & Per day & {$[66]$} \\
\hline$k_{2}$ & $0.1-1.0$ & Per day & [65] & $\eta_{1}$ & 0.07 & Per day & {$[8,64]$} \\
\hline$\varphi(v)$ & - & - & - & $\eta_{2}$ & 0.07 & Per day & [66] \\
\hline$C_{m}$ & 95 & $\mathrm{mmHg}$ & {$[8,64]$} & $a$ & 0.1 & - & [64] \\
\hline$\gamma_{1}$ & 0.8 & Per day & {$[66]$} & $b$ & 2.0 & - & {$[8,64]$} \\
\hline$\gamma_{2}$ & 0.4 & Per day & {$[66]$} & $c_{r}$ & 0.05 & - & [8] \\
\hline$\alpha$ & 0.06 & Per day & {$[8,64]$} & $c_{m}$ & 0.05 & - & {$[8,64]$} \\
\hline
\end{tabular}

Table 2. Initial parameter values for breast cancer progression in the model.

Note that one microvessel unit (MU) is equal to the mean length of microvessel in one gram of unaffected cell or tissue. 


\section{Results and Discussions}

The system of the differential equations was numerically solved using MATLAB using the parameter values summarized in Table 2. Results from the model are in silico, meaning that they can be applied to a clinical setting. Here, the term "injected vaccine" refers to computerized simulation of a host's immune response after inoculated with a vaccine, and then subsequently introduced into the tumor. The timescale of the figures was utilized to provide the basis for how the model can be applied to a clinical setting.

In Figure 2 we showed that if there's no cancer vaccine injected, a host produced an immune response that is not sufficient to eliminate the cancer tumor. In this regard, it was observed that a cancer cells with a sufficiently large VEC density was able to produce a viable cancer. As a result, the cancer cells, the VEC, and the microvessel with standard parameter values and initial conditions grew exponentially, as shown in Figures 2 and 3. The mass of the cancer cells in particular reached a mass of $28 \mathrm{~g}$ in 190 days.

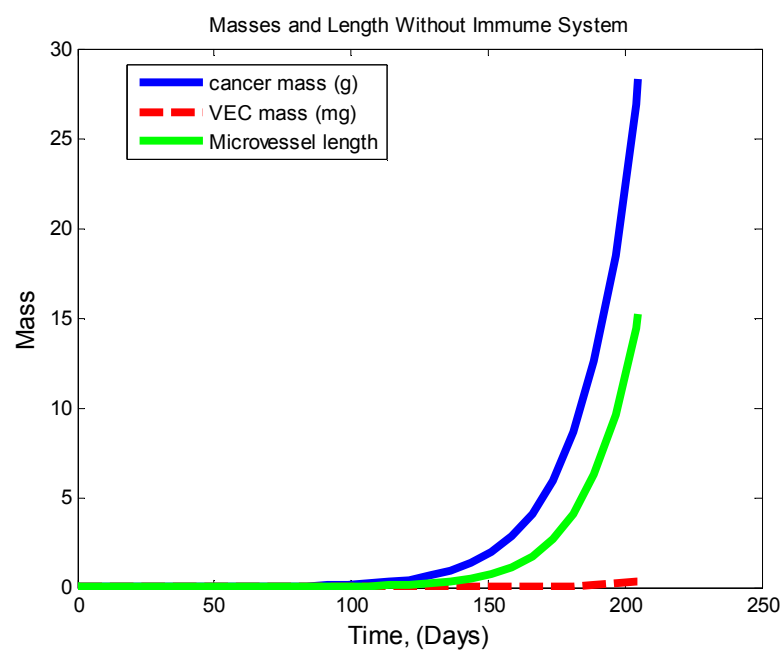

Figure 2. A viable breast cancer tumor when no cancer vaccine is injected.

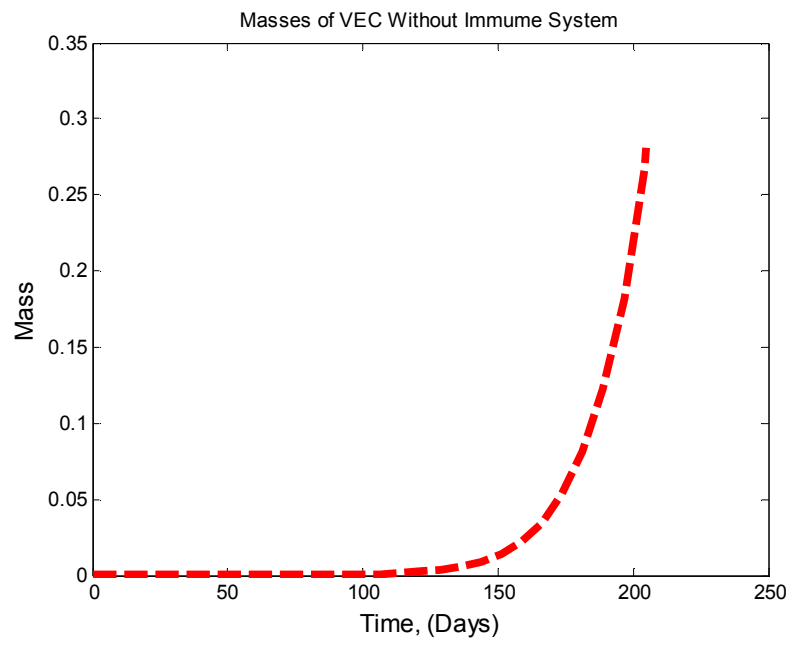

Figure 3. A sufficiently large VEC density increasing exponential to promote a viable cancer.
Once the injected immune vaccine was introduced, the maximum mass of breast cancer cells reached only 6.2 grams in approximately 120 days (see Figure 4). In addition, both VEC mass and the microvessel length reduced to $0.075 \mathrm{mg}$ and 5 MU respectively, as shown in Figure 5. These observations indicated that both VEC mass and microvessel density within the regions of the cancer were prognostic indicators for the human breast carcinoma.

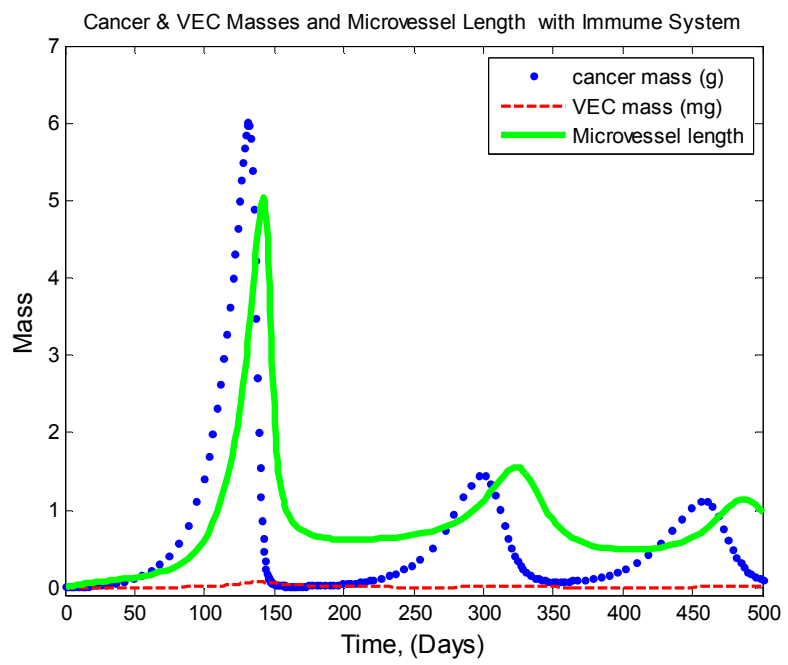

Figure 4. When cancer vaccine is injected and immune system parameters activated resulted in positive correlation between the microvessel density length and the breast cancer growth.

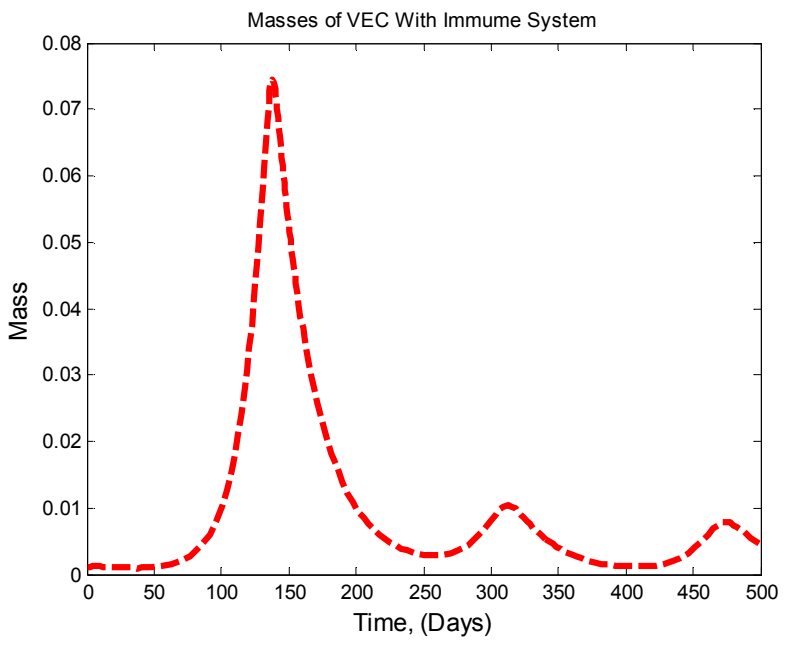

Figure 5. The mass of VEC with immune system is plotted against number of days.

At the inflection point of the cancer's growth curve, macrophages, NK cells and helper T cells began to respond well to the cancer while the CTLs were also activated at the same time, see Figure 6. Further analysis on CTLs and helper $\mathrm{T}$ cells showed that a sufficiently high rate of infiltration of either cells reduced tumor growth. If the rate of immune infiltration of helper $\mathrm{T}$ cells was low, a large rate of CTLs cells infiltration was needed to eliminate the tumor. On the other hand, if the CTLs cells infiltration was low, only a 
moderate increase in the helper $\mathrm{T}$ cell population was required to eradicate the tumor. Similarly, tumor-infiltrating macrophages and NK cells also depicted similar results. Thus, the combined effect of the immune response reduced the cancer cells drastically. In addition, after the cancer cells, VEC, and the microvessel density reached their peaks, the cancer cell mass continued to oscillate in what appeared to be a stable limit cycle. Optimizing the values of the four immune elements caused the cancer cells to decrease in mass and relapse time, as shown in Figure 7.

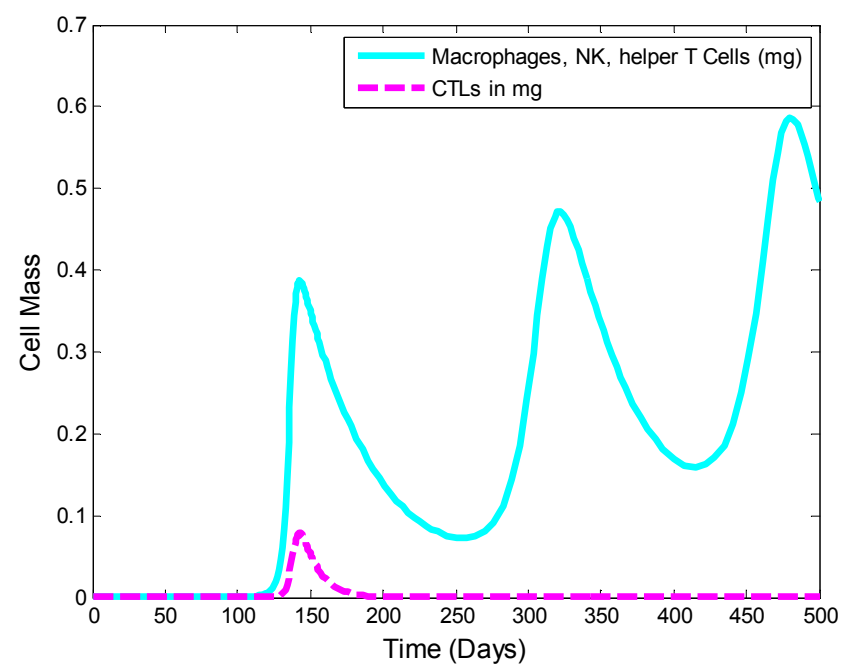

Figure 6. Graph of macrophages, natural killer, helper T cells and CTLs masses plotted against time in days shows immune activations.

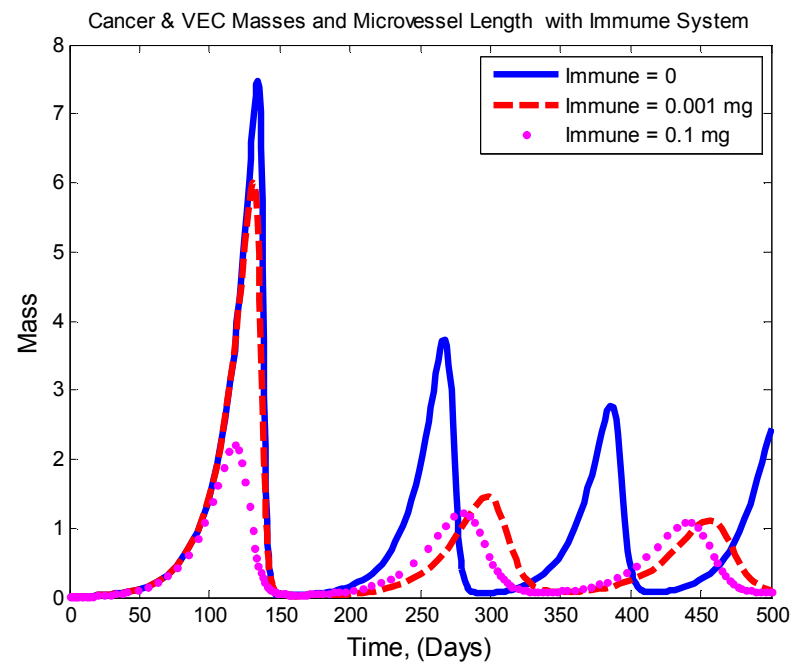

Figure 7. The effects of different sizes of immunity on cancer cells.

To analyze the model mechanism by which macrophages, NK, and helper T cells were able to activate CTLs, we varied their activation rates in presence of cancer cells $\left(\lambda_{a}\right)$ from 0.05 to 0.5 . The plot showed a chaotic behavior as shown in Figure 8 . The chaotic nature of the cancer growth might be attributed to possible time-delays introduced by immunemediated activation of CTLs and helper T cells or/and the controversy related to large numbers of tumor-infiltrating macrophages (the M1 and M2). The importance of large
M1:M2 ratio verses large/small numbers of tumor-infiltrating macrophages on long-term cancer patient could have some impact on this behavior. In addition, a huge CTL activation spike ( $\sim 2800 \mathrm{mg}$ ) was observed between 1500-1580 days, see Figure 9. What exactly caused the abnormally high CTLs spike under very low levels of macrophages, NK and helper $\mathrm{T}$ cells remained unclear. However, there were large time spans between the cancer decay and regrowth during the peak activation of the CTLs. Since $\lambda_{a}$ represented the activation rates of immune cells that participated in direct killing of cancer cells and also drove the activation of CTLs, we could deduce that increasing $\lambda_{a}$ would help eliminate the cancer cell.

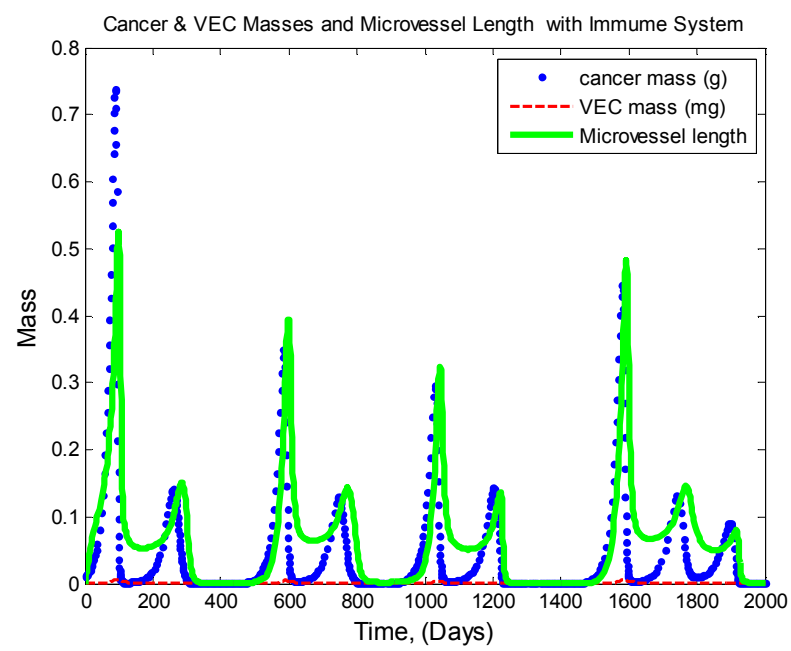

Figure 8. The effect in which macrophages, Natural Killer cells and helper T cells activates the CTLs in presence of breast cancer.

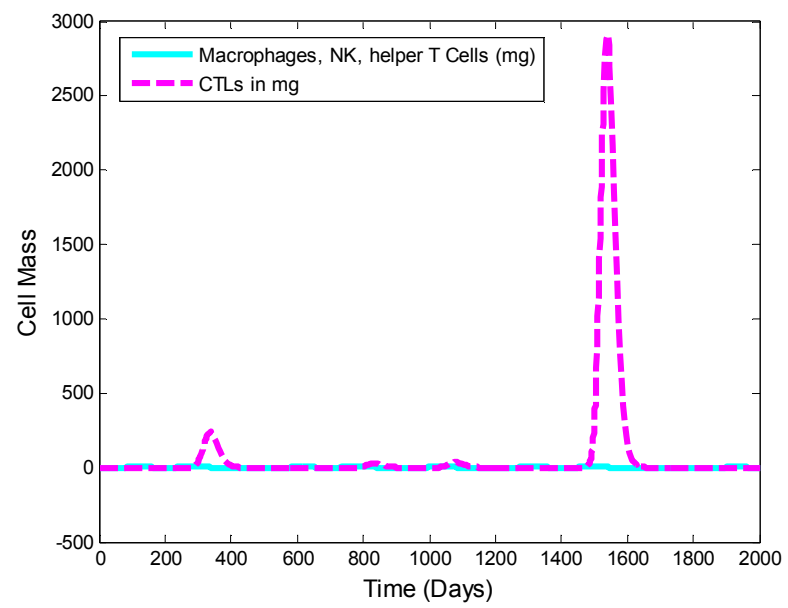

Figure 9. Graph of CTLs activation behavior when cancer size was increased.

The rate at which macrophages, NK, and helper $\mathrm{T}$ cells $\left(k_{1}\right)$ and CTLs $\left(k_{2}\right)$ killed breast cancer were represented in Figure 10. By setting the CTLs cells $(n)$ to zero, we observed that as $k_{1}$ increases a stable oscillatory behavior and a much less cancer mass were reported indicating that $k_{1}$ 
effectively kills the cancer cells. Varying $k_{2}$ by setting the stimulatory immune cells capable of attaching cancer cells (i.e. $m$ ) to zero resulted in the activation of CTLs and stimulatory immune cells with a stable limit cycle able to remove cancer efficiently. Similar observation was discovered when $k_{2}>k_{1}$ and setting the cancer-mediated CTL activation rate to zero.

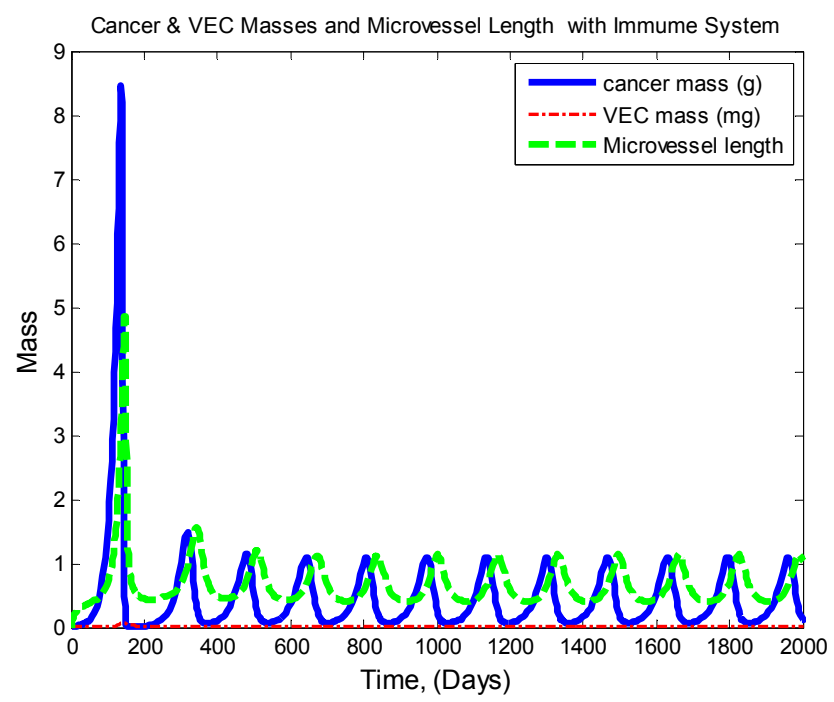

Figure 10. Effect of optimal CTLs activation on cancer cells, VEC and microvessel.

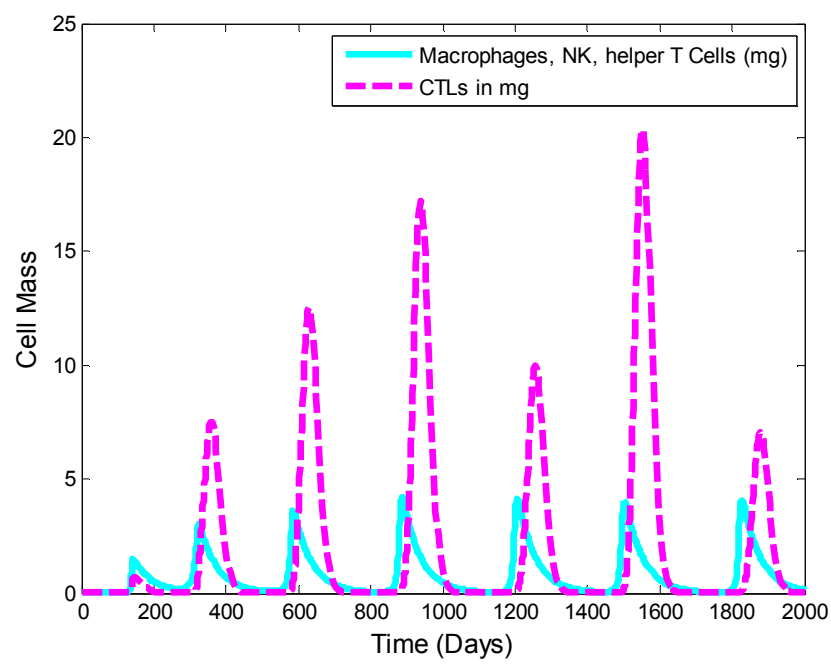

Figure 11. Effects of CTLs, macrophages, natural killers, and helper T cells on cancer cell mass.

The immune cells and CTLs response graph is depicted in Figure 11. As observed, the CTL activation overshadowed and coincided with the peaks of macrophage, NK and T cell activation when cancer cells were present. As the cancer size increased, there was a strong interaction between the cancer cells and CTLs which increased the cancer cell and CTLs ratio. Steadily, this interaction killed enough cancer cells to cause the decrease in cancer size. However, this increased the CTL suppression which decreased the regulating of the CTL activities. Consequently, the cancer cells start growing again only to reactivate CTLs with much larger value than the one obtained during the previous peak. This observation further supports the fact that the immune-mediated activation of CTLs is a major factor in developing anti breast cancer immunity.

\section{Conclusion}

We applied mathematical modeling as a tool to depict the relative strength of the host's immune system after it has been subjected to a breast cancer vaccine consisting of CTLs, helper T, Macrophages, and NK cells. The results supported the hypothesis that the stimulatory roles of macrophages, natural killer cells and helper T-cells play an important role in establishing an effective anti-cancer response of breast cancer growth. Specifically, we observed that breast cancer size was reduced drastically when the immune vaccines were activated. Given a sufficiently high rate of CTLs or helper $\mathrm{T}$ cells infiltration responded with tumor elimination. If the rate of immune infiltration of helper $\mathrm{T}$ cells was low, a large rate of CTLs cells infiltration was needed to eliminate the tumor. On the other hand, if the CTLs cells infiltration was low, only a moderate increase in the helper $\mathrm{T}$ cell population was required to eradicate the tumor. In addition, we also observed the importance of large M1:M2 ratios verses large/small numbers of tumor-infiltrating macrophages on long term patient survival. Even though the combined effect allow us to make predictions on long-term survival, it is necessary to optimize both numbers and percentages of M1 and M1 cells used with tumor-infiltrating macrophages in the context of improving breast cancer therapies. Thus, the assessment of immune activation could improve prognostic stratifications of $\mathrm{T}$ cell subsets (CTLs and helper T cells) and macrophages (M1 and M2) and therefore could be an effective therapies in cancer treatment. Furthermore, breast cancer size with different growth rates provoked different immune responses. Thus, for a given set of immune system parameters, the effectiveness of the immune system against breast cancer depended on the cancer growth rate and the immunity response. Hence, given the evidence discussed in this paper, the hypothesis that immune system status is implicated in breast cancer relapse or cancer prevention deserves consideration and further investigation. The integration of immunotherapy into the management of breast cancer is challenging in experimental and clinical settings. Vaccines can elicit an antigen specific immune response, but the immunity discussed are complex and tends to fade with time. Mathematical modeling approach can provide or improve our knowledge of the interactions between vaccine therapies, breast carcinoma, and immunity.

\section{References}

[1] Siegel, RL, Miller, KD, Jemal, A. Cancer statistics, 2019. CA Cancer J Clin. 2019; 68:7-30. doi:10.3322/caac.21442. 
[2] Loi S. Tumor-infiltrating lymphocytes, breast cancer subtypes and therapeutic efficacy. Oncoimmunology. 2013; 2(7):e24720.

[3] Heidi Dritschel, Sarah L. Waters, Andreas Roller \& Helen M. Byrne (2018). A mathematical model of cytotoxic and helper $\mathrm{T}$ cell interactions in a tumour microenvironment, Letters in Biomathematics, 5:sup1, S36-S68, DOI: $10.1080 / 23737867.2018 .1465863$

[4] Denkert C, Loibl S, Noske A, et al. Tumor-associated lymphocytes as an independent predictor of response to neoadjuvant chemotherapy in breast cancer. $J$ Clin Oncol. $2010 ; 28(1): 105-113$

[5] Gu-Trantien C, Loi S, Garaud S, et al. $\mathrm{CD}^{+}$follicular helper $\mathrm{T}$ cell infiltration predicts breast cancer survival. J Clin Invest. 2013; 123(7):2873-2892.

[6] Loi S, Sirtaine N, Piette F, et al. Prognostic and predictive value of tumor-infiltrating lymphocytes in a phase III randomized adjuvant breast cancer trial in nodepositive breast cancer comparing the addition of docetaxel to doxorubicin with doxorubicin-based chemotherapy: BIG 02-98. J Clin Oncol. 2013; 31(7):860-867.

[7] Loi S. Tumor-infiltrating lymphocytes, breast cancer subtypes and therapeutic efficacy. Oncoimmunology. 2013; 2(7):e24720.

[8] Quinonez J, Dasu N, Qureshi M. A Mathematical Investigation on Tumor-Immune Dynamics: The Impact of Vaccines on the Immune Response. J Cancer Sci Ther 2017 9: 675-682. doi:10.4172/1948-5956.1000491

[9] Benedetti, R., Dell'Aversana, C., Giorgio, C., Astorri, R., \& Altucci, L. (2017). Breast Cancer Vaccines: New Insights. $\begin{array}{llll}\text { Frontiers in endocrinology, 8, } 270 . & \text {. }\end{array}$ doi:10.3389/fendo.2017.00270.

[10] Baraka A, Borai M, Hesam M, Almalky M. Study of Tregulatory cells in patients with acute, idiopathic thrombocytopenic purpura. Egyptian J Haematol. 2014 39: 37.

[11] Gu-Trantien C, Loi S, Garaud S, et al. $\mathrm{CD}^{+}$follicular helper $\mathrm{T}$ cell infiltration predicts breast cancer survival. J Clin Invest. 2013; 123(7):2873-2892.

[12] Fridman WH, Galon J, Pages F, Tartour E, Sautes-Fridman C, Kroemer G. Prognostic and predictive impact of intra- and peritumoral immune infiltrates. Cancer Res. 2011; 71(17): 5601-5605.

[13] Slichter SJ, Bolgiano D, Corson J, Jones MK, Christoffel T. Extended storage of platelet-rich plasma-prepared platelet concentrates in plasma or Plasmalyte. Transfusion. 2010; 50(10):2199-2209.

[14] West NR, Milne K, Truong PT, Macpherson N, Nelson BH, Watson PH. Tumor-infiltrating lymphocytes predict response to anthracycline-based chemotherapy in estrogen receptornegative breast cancer. Breast Cancer Res. 2011; 13(6):R126.

[15] Yamaguchi R, Tanaka M, Yano A, et al. Tumor-infiltrating lymphocytes are important pathologic predictors for neoadjuvant chemotherapy in patients with breast cancer. Hum Pathol. 2012; 43(10):1688-1694.

[16] Lee HJ, Seo J-Y, Ahn J-H, Ahn S-H, Gong G. Tumorassociated lymphocytes predict response to neoadjuvant chemotherapy in breast cancer patients. $J$ Breast Cancer.
$2013 ; 16(1): 32-39$

[17] Ono M, Tsuda H, Shimizu C, et al. Tumor-infiltrating lymphocytes are correlated with response to neoadjuvant chemotherapy in triple-negative breast cancer. Breast Cancer Res Treat. 2012; 132(3):793-805.

[18] Issa-Nummer Y, Darb-Esfahani S, Loibl S, et al. Prospective validation of immunological infiltrate for prediction of response to neoadjuvant chemotherapy in HER2-negative breast cancer - a substudy of the neoadjuvant GeparQuinto trial. PLoS One. 2013; 8(12):e79775.

[19] Salgado R, Denkert C, Demaria S, et al. The evaluation of tumor-infiltrating lymphocytes (TILs) in breast cancer: recommandations by an international TILs working group 2014. Ann Oncol. 2015; 26(2):259-271.

[20] Ono M, Tsuda H, Shimizu C, et al. Tumor-infiltrating lymphocytes are correlated with response to neoadjuvant chemotherapy in triple-negative breast cancer. Breast Cancer Res Treat. 2012; 132(3):793-805.

[21] Issa-Nummer Y, Darb-Esfahani S, Loibl S, et al. Prospective validation of immunological infiltrate for prediction of response to neoadjuvant chemotherapy in HER2-negative breast cancer - a substudy of the neoadjuvant GeparQuinto trial. PLoS One. 2013; 8(12):e79775.

[22] Salgado R, Denkert C, Demaria S, et al. The evaluation of tumor-infiltrating lymphocytes (TILs) in breast cancer: recommandations by an international TILs working group 2014. Ann Oncol. 2015; 26(2):259-271.

[23] Ladoire S, Arnould L, Apetoh L, et al. Pathologic complete response to neoadjuvant chemotherapy of breast carcinoma is associated with the disappearance of tumor-infiltrating foxp3 regulatory T cells. Clin Cancer Res. 2008; 14(8):2413-2420.

[24] Hodi FS, O’Day SJ, McDermott DF, et al. Improved survival with ipilimumab in patients with metastatic melanoma. $N$ Engl J Med. 2010; 363(8):711-723.

[25] Loi S, Michiels S, Salgado R, et al. Tumor infiltrating lymphocytes are prognostic in triple negative breast cancer and predictive for trastuzumab benefit in early breast cancer: results from the FinHER trial. Ann Oncol. 2014; 25(8):15441550 .

[26] Adams S, Gray RJ, Demaria S, et al. Prognostic value of tumor-infiltrating lymphocytes in triple-negative breast cancers from two phase III randomized adjuvant breast cancer trials: ECOG 2197 and ECOG 1199. J Clin Oncol. 2014; 32(27):2959-2966.

[27] Dieci MV, Criscitiello C, Goubar A, et al. Prognostic value of tumor-infiltrating lymphocytes on residual disease after primary chemotherapy for triple-negative breast cancer: a retrospective multicenter study. Ann Oncol. 2014; 25(3):611-618.

[28] Azim HA Jr, Vingiani A, Peccatori F, Viale G, Loi S, Pruneri G. Tumour infiltrating lymphocytes (TILs) in breast cancer during pregnancy. Breast. 2015; S0960- 9776(15)00010-7.

[29] Nagalla S, Chou JW, Willingham MC, et al. Interactions between immunity, proliferation and molecular subtype in breast cancer prognosis. Genome Biol. 2013; 14(4):R34.

[30] Mittendorf EA, Peoples GE. Injecting hope - a review of breast cancer vaccines. Oncology (Williston Park) (2016) 30(5):47581 . 
[31] Perez EA, Thompson EA, Ballman KV, et al. Genomic analysis reveals that immune function genes are strongly linked to clinical outcome in the north central cancer treatment group n9831 adjuvant trastuzumab trial. J Clin Oncol. 2015; 33(7):701-708.

[32] Adnan Aydiner, Systemic Treatment of HER2-Overexpressing Metastatic Breast Cancer, Breast Cancer, 10.1007/978-3-31996947-3_23, (509-531), (2018).

[33] Cui N, Shi J, Yang C. HER2-Based Immunotherapy for Breast Cancer. Cancer Biother Radiopharm. 2018 Jun; 33(5):169175. Epub 2018 Jun 6.

[34] Seliger B, Steven A: The role of immune escape and immune cell infiltration in breast cancer. Breast Care 2018; 13:DOI:10.1159/000486585.

[35] Schütz F, Marmé F, Domschke C, Sohn C, von Au A: Immunooncology in breast cancer: active and passive vaccination strategies. Breast Care 2018; 13:DOI: $10.1159 / 000486330$

[36] Lehmann BD, Bauer JA, Chen X, et al. Identification of human triple-negative breast cancer subtypes and preclinical models for selection of targeted therapies. J Clin Invest. 2011; 121(7):2750-2767.

[37] Gu-Trantien C, Loi S, Garaud S, et al. $\mathrm{CD}^{+}$follicular helper $\mathrm{T}$ cell infiltration predicts breast cancer survival. J Clin Invest. 2013; 123(7):2873-2892.

[38] Hiraoka, K. , Miyamoto, M. , Cho, Y. , Suzuoki, M. , Oshikiri, T. , Nakakubo, Y. , Katoh, H. Concurrent infiltration by CD8+ $\mathrm{T}$ cells and CD4+ $\mathrm{T}$ cells is a favourable prognostic factor in non-small-cell lung carcinoma. British Journal of Cancer, 2006, 94 (2), 275-280.

[39] Rad, F. , Ajdary, S. , Omranipour, R. , Alimohammadian, M. ,\& Hassan, Z. Comparative analysis of CD4+ and CD8+ T cells in tumor tissues, Lymph nodes and the peripheral blood from patients with breast cancer. Iranian Biomedical Journal, $2015,19(1), 35$.

[40] Badoual, C. , Hans, S. , Rodriguez, J. , Peyrard, S. , Klein, C. , Agueznay, N. E. H. , ... Gey, A. Prognostic value of tumorinfiltrating CD4+ T-cell subpopulations in head and neck cancers. Clinical Cancer Research. 2006, 12 (2), 465-472.

[41] Magombedze, G. , Reddy, P. , Eda, S. , \& Ganusov, V. Cellular and population plasticity of helper $\mathrm{CD} 4+\mathrm{T}$ cell responses. Frontiers in Physiology. 2013, 4, 206.

[42] Fridman, W. H. , Pages, F. , Sautes-Fridman, C. , \& Galon, J. The immune contexture in human tumours: Impact on clinical outcome. Nature Reviews Cancer. 2012, 12 (4), 298-306.

[43] Chen, D. S. , \& Mellman, I.(2013). Oncology meets immunology: The cancer-immunity cycle. Immunity. 2013, 39(1), 1-10.

[44] Magombedze, G. , Reddy, P., Eda, S. , \& Ganusov, V. (2013). Cellular and population plasticity of helper CD4+ T cell responses. Frontiers in Physiology. 2013, 4, 206.

[45] Cassetta, L., Cassol, E., \& Poli, G. Macrophage polarisation in health and disease. TheScientificWorld JOURNAL. 2011, 11, 2391-2402.

[46] Italiani, P., \& Boraschi, D. From monocytes to M1/M2 macrophages: Phenotypical vs. functional differentiation.
Frontiers in Immunology. 2014, 5, 514.

[47] Jakeman, P. G., Hills, T. E., Fisher, K. D., \& Seymour, L. W. (2015). Macrophages and their interactions with oncolytic viruses. Current Opinion in Pharmacology. 2015, 24, 23-29.

[48] Denton, N. L., Chen, C.-Y., Scott, T. R., \& Cripe, T. P. Tumour-associated macrophages in oncolytic virotherapy: Friend or foe? Biomedicines. 2016, 4, 13.

[49] Hamilton G., Reiner A., Teleky B. et al. Natural killer cell activities of patients with breast cancer against different target cells. J Cancer Res Clin Oncol. (1988) 114:191-196.

[50] Diefenbach A. and Raulet D. H. The innate immune response to tumors and its role in the induction of T-cell immunity, Immunological Reviews. 2002, Vol. 188 Issue1, pp 9-21.

[51] Orange J. S. Human Natural Killer cell defiencies. Curr Opin Allergy Clin Immunol, 6:(6): (2006) 399-409.

[52] Berrien-Elliot MM, Romee R, Fehniger TA. Improving natural killer cell cancer immunotherapy. Curr Opin Organ Transplant (2015) 20(6):671-80.10.1097/MOT.0000000000000243

[53] Pillis L. G. and Radunskaya A. A Mathematical Tumor Model with Immune Resistance and Drug Therapy: an Optimal Control Approach. Journal of Theoretical Medicine, (2000), Vol. 3, 79-100.

[54] Eisen M. Mathematical models in cell biology and cancer chemotherapy. Berlin: Springer -Verlag; Berlin, 1979.

[55] Bellomo N., Preziosi L. Modelling and mathematical problems related to tumor evolution and its interaction with the immune system. Mathematical and Computer Model ling (2000) 32:41 3-52.

[56] Coldman A. J., Goldie J. H. A stochastic model for the origin and treatment of tumors containing drug-resistant cells. Bull Math Biol. 1986; 48:27 9-92.

[57] Eisen M. Mathematical models in cell biology and cancer chemotherapy. Berlin: Springer -Verlag; Berlin, 1979.

[58] Bellomo N., Preziosi L. Modelling and mathematical problems related to tumor evolution and its interaction with the immune system. Mathematical and Computer Model ling (2000) 32:41 3-52.

[59] Coldman A. J., Goldie J. H. A stochastic model for the origin and treatment of tumors containing drug-resistant cells. Bull Math Biol. 1986; 48:27 9-92.

[60] Skipper H. E. On mathematical modeling of critical variables in cancer treatment (Goals: better understanding of the past and better planning in the future). Bull Math Biol. 1986; 48:25 3-78.

[61] Rubinstein W. S., O'Neill S. M., Peters J. A., Rittmeyer L. J., Stadler M. P. Mathematical modeling for breast cancer risk assessment. State of the art and role in medicine. Oncology, (2002), 16(8):1082-94; discussion 1094, 1097-9.

[62] Roe-Dale R., Isaacson D., Kupferschmid M. A Mathematical Model of Breast Cancer Treatment with CMF and Doxorubicin. Bull Math Biol. (2011) 73: 585-608

[63] d'Onofrio A. A general framework for modeling tumorimmune system competition and immunotherapy: Mathematical analysis and biomedical inferences, Physica D 208 (2005) 220-235 
[64] Nagy J. D. Competition and Natural Selection in a Mathematical Model of Cancer. Bulletin of Mathematical Biology, (2004), 66, 663-687.

[65] Thomas D. and Massague J. TGF- $P$ directly targets cytotoxic T-cell functions during tumor evasion of immune surveillance Cancer cell 8, (2005) 369-380.
[66] De Pillis LG, Radunskaya AE, Wiseman CL. A validated mathematical model of cell mediated immune response to tumor growth. Cancer Res 2005, 65: 7950-7958. 